\title{
Formation of Pyrene Excimers in Mesoporous Ormosil Thin Films for Visual Detection of Nitro-explosives
}

\author{
Pinar Beyazkilic, ${ }^{\dagger, \ddagger}$ Adem Yildirim, ${ }^{\dagger, \ddagger}$ and Mehmet Bayindir ${ }^{*} \dagger, \ddagger, \S$ \\ ${ }^{\dagger}$ UNAM-National Nanotechnology Research Center, ${ }^{\ddagger}$ Institute of Materials Science and Nanotechnology, and ${ }^{\S}$ Department of \\ Physics, Bilkent University, 06800 Ankara, Turkey
}

\section{Supporting Information}

ABSTRACT: We report the preparation of mesoporous thin films with bright pyrene excimer emission and their application in visual and rapid detection of nitroaromatic explosive vapors. The fluorescent films were produced by physically encapsulating pyrene molecules in the organically modified silica (ormosil) networks which were prepared via a facile template-free sol-gel method. Formation and stability of pyrene excimer emission were investigated in both porous and nonporous ormosil thin films. Excimer emission was significantly brighter and excimer formation ability was more stable in porous films compared to nonporous films. Rapid and selective quenching was observed in the excimer emission against vapors of nitroaromatic molecules; trinitrotoluene (TNT), dinitrotoluene (DNT), and nitrobenzene (NB). Fluorescence quenching of the films can be easily observed under UV light, enabling the naked-eye detection of nitro-explosives. Furthermore, excimer emission signal can be recovered after quenching and the films can be reused at least five times.

KEYWORDS: pyrene, excimer emission, porous thin film, organically modified silica, nitro-explosive detection

\section{INTRODUCTION}

Pyrene and its derivatives, with their high quantum yield and chemical stability, are well-studied fluorophores for molecular labeling and fluorescent sensing applications. ${ }^{1,2}$ Pyrene has two main emission bands; first one is the monomer emission of pyrene (i.e., distance between molecules is greater than $\sim 1 \mathrm{~nm}$ ) between 370 and $400 \mathrm{~nm}$ and second is the excimer emission of pyrene (i.e., distance is less than $\sim 1 \mathrm{~nm}$ ) at around $470 \mathrm{~nm}$., The former occurs when a single pyrene molecule is excited by light and relaxes back to its ground state; the latter occurs when a pyrene molecule in its excited state interacts with nearby ground-state pyrene molecule and forms an excited dimer at a lower electronic energy state than that of a single excited pyrene. 3,4

The bright and visible pyrene excimer emission is particularly interesting because it is very sensitive to the microenvironmental conditions such as temperature, pressure, or $\mathrm{pH} .{ }^{1}$ In addition, excimer emission can be affected by guest molecules and accordingly it can be used to detect various classes of chemicals including gases, ${ }^{5,6}$ organic molecules, ${ }^{7,8}$ and metal ions. ${ }^{9,10}$ In particular, pyrene excimer emission can be rapidly quenched via exposure to nitroaromatic explosives based on electron-transfer between $\pi-\pi^{*}$ stacked pyrene molecules and nitroaromatic molecules. ${ }^{11-13}$ Therefore, thin films with excimer emission are very promising materials as fluorescence sensors for numerous vapor phase analytes. However, preparation of thin films with strong pyrene excimer emission is challenging due to the loose interaction between pyrene molecules after solvent evaporation. ${ }^{14}$ Most preferred method to prevent decay of excimer emission is the covalent attachment of pyrene moieties to the thin film network. ${ }^{15}$ Nevertheless, it was reported that poor excimer emission signal can be observed even for the covalently bonded pyrene molecules. ${ }^{16}$ Furthermore, covalent attachment of pyrene derivatives requires tedious and costly organic synthesis steps. Another method is the physical encapsulation of pyrene fluorophores during thin film preparation. ${ }^{12,13,15}$ However, in order to observe excimer emission, polymers such as polystyrene (PS) or polyethersulfone (PES), which are structurally similar to pyrene must be used. In most of the studies, strong excimer emission is observed by using thin films prepared from these polymers. However, time-dependent excimer formation ability is not considered in these studies. ${ }^{12,13,17,18}$ In fact, decay of pyrene excimer emission has been frequently used to evaluate the structure of sol-gel materials. ${ }^{19-22}$ Besides poor excimer formation, another drawback of physical encapsulation method is the utilization of dense polymers as thin film networks. Analyte diffusion through the dense polymer matrix takes very long times and therefore sensing performances of films decrease. $^{23-25}$ Very thin $(<100 \mathrm{~nm})$ films can be prepared in order to eliminate this effect; however, measurements with very thin films are not reliable and repeatable due to low fluorescence signal of these films. ${ }^{15}$ Apparently, porous thin films are more suitable than their nonporous counterparts for vapor phase fluorescent sensing applications, where transport

Received: December 30, 2013

Accepted: March 17, 2014

Published: March 17, 2014 
rate of analytes through the porous network is higher and therefore fluorescence response is faster. ${ }^{23-27}$

Herein, we report formation of pyrene excimers in mesoporous organically modified silica (ormosil) thin films using physical encapsulation method. We prepared ormosil thin films via a facile template-free sol-gel method and we physically encapsulated pyrene molecules in the ormosil network during synthesis without making any chemical modification to pyrene. We observed that pyrene excimer formation in porous ormosil films was more promoted and excimer emission was stronger compared to their nonporous counterparts. Strong excimer emission in porous materials was also previously observed by mixing pyrenes with porous cellulose $\mathrm{e}^{28}$ and by chemically attaching to mesoporous silica surfaces. $^{29}$ In addition, we analyzed nitroaromatic explosive sensing performances of porous thin films for trinitrotoluene (TNT), dinitrotoluene (DNT) and nitrobenzene (NB) based on fluorescence quenching. It was observed that fluorescence quenching efficiencies were as high as 32 and $87.4 \%$ for TNT after $10 \mathrm{~s}$ and $5 \mathrm{~min}$, respectively. In our previous work, we encapsulated a porphyrin derivative into ormosil thin film as fluorescent sensing molecule. Quenching efficiency of that film was only $8.6 \%$ for TNT after $10 \mathrm{s.}^{27}$ In addition to the improved quenching efficiency in the present study, the rapid fluorescence quenching of porous ormosil films can be observed visually under UV light (Scheme 1) without the

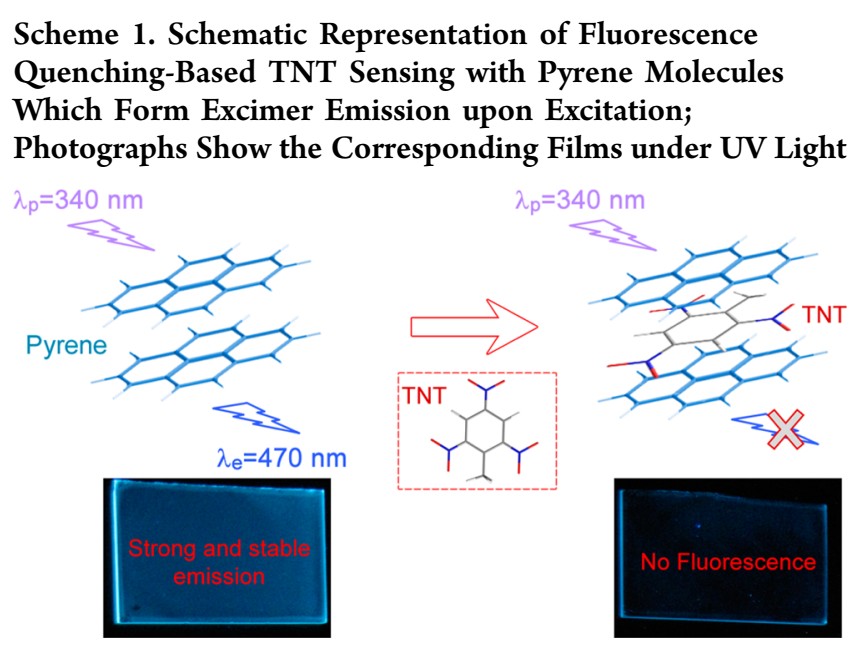

need of any complicated equipment. The selectivity of the sensor against nitroaromatic compounds was demonstrated by using vapors of various aromatic and nonaromatic molecules. Furthermore, excimer emission signal of the porous ormosil films can be largely recovered by simply washing with water after quenching. Finally, we showed that the films are reusable at least five times.

\section{EXPERIMENTAL SECTION}

Materials. Methyltrimethoxysilane (MTMS), dimethylsulfoxide (DMSO), methanol, oxalic acid, and ammonium hydroxide (25\%) were purchased from Merck (Germany). Ethanol, hydrochloric acid (37\%), and pyrene were purchased from Sigma-Aldrich. All chemicals were used as received without any purification.

Preparation of Pyrene Doped Mesoporous Ormosil Thin Films. Ormosil thin films were prepared by modifying the previous methods. ${ }^{27,30-32}$ Ormosil gel was first prepared via a two-step sol-gel reaction (see Scheme S1 in the Supporting Information for the experimental steps). First, $1 \mathrm{~mL}$ of MTMS was added to the mixture containing $4.88 \mathrm{~mL}$ of methanol and $3.88 \mathrm{~mL}$ of DMSO. Different amounts of pyrene was dissolved in $1 \mathrm{~mL}$ of DMSO and added to MTMS solutions. For example, $20 \mathrm{mg}$ of pyrene was used to obtain a gel containing $8.34 \mathrm{mM}$ pyrene. Then, $500 \mu \mathrm{L}$ of $1.0 \mathrm{mM}$ aqueous oxalic acid solution was added dropwise to start the hydrolysis of monomer. Synthesis solution was stirred for $30 \mathrm{~min}$ and then was left to hydrolyze completely for $24 \mathrm{~h}$ at room temperature. After the hydrolysis step, $0.42 \mathrm{~mL}$ of ammonium hydroxide (25\%) solution and $0.19 \mathrm{~mL}$ of deionized water were added to the solution and the solution was stirred for further $15 \mathrm{~min}$. Finally, the solution was poured into a polystyrene vial and left for gelation at $25{ }^{\circ} \mathrm{C}$. The gel was aged at this temperature for $48 \mathrm{~h}$ in order to strengthen the porous network. After aging, methanol was added onto gel and the gel was sonicated by using an ultrasonic liquid homogenizer at $20 \mathrm{~W}$ for $45 \mathrm{~s}$. Films with three different thicknesses were prepared by diluting the ormosil gels, which were containing $8.34 \mathrm{mM}$ pyrene, with different amounts of methanol. For example, $12 \mathrm{~mL}$ of methanol added onto gel for $251 \mathrm{~nm}$ thick film. Finally, an ormosil sol which is suitable for thin film deposition was obtained. Because of the dilution with methanol, pyrene concentration in the ormosil colloids decreased from 8.34 to $4.14 \mathrm{mM}$. Glass substrates with $2 \mathrm{~cm} \times 1 \mathrm{~cm}$ dimensions were cut, then cleaned in ethanol and isopropanol mixture and finally dried, and $250 \mu \mathrm{L}$ portions of the ormosil sol were dispensed onto glass substrates and coated by spin-coating at $3000 \mathrm{rpm}$ for $45 \mathrm{~s}$. Most of the solvent evaporated and the final film structure established during the spin-coating process.

Preparation of Pyrene Doped Nonporous Ormosil Films. We prepared the nonporous films by slightly modifying our previous method. ${ }^{27}$ First, $2.25 \mathrm{~mL}$ of MTMS was dissolved in $1.12 \mathrm{~mL}$ of ethanol. Twenty milligrams of pyrene was dissolved in $1.12 \mathrm{~mL}$ of DMSO. Then, pyrene solution, $0.2 \mathrm{~mL}$ of deionized water and $5 \mu \mathrm{L}$ of $0.1 \mathrm{M}$ hydrochloric acid aqueous solution were added under gentle stirring. Synthesis solution was stirred at $60{ }^{\circ} \mathrm{C}$ for $90 \mathrm{~min}$. After that, $0.4 \mathrm{~mL}$ of $0.1 \mathrm{M}$ hydrochloric acid aqueous solution and $0.35 \mathrm{~mL}$ of deionized water were added and the solution was further stirred at ambient temperature for $15 \mathrm{~min}$. Finally, the sol was left for aging at 50 ${ }^{\circ} \mathrm{C}$ for $15 \mathrm{~min}$. Two milliliters of the aged sol was diluted with $0.4 \mathrm{~mL}$ of methanol in order to adjust the film thickness around $275 \mathrm{~nm} .250$ $\mu \mathrm{L}$ portions of the sol were dispensed onto previously cleaned glass substrates and were coated by spin-coating at $3000 \mathrm{rpm}$ for $45 \mathrm{~s}$.

Nitro-Explosive Sensing Experiments. Approximately $10 \mathrm{mg}$ of TNT was put inside a $15 \mathrm{~mL}$ vial and then a piece of cotton was placed onto top to prevent direct contact of films with TNT. Vial was kept closed at least for 2 days prior to sensing experiments in order to ensure that TNT vapor reached equilibrium. Sensing experiments were performed at room temperature where vapor pressure of TNT is 5 $\mathrm{ppb}^{33}$ Before exposing the spin-coated porous film to TNT vapor, emission of the film was recorded with fluorescence spectrophotometer. Then, film was placed onto the cotton piece in the vial and kept closed for a specific time. After that the film was taken out of the vial and its emission was recorded again. Samples of DNT, benzoic acid, ammonium nitrate and 3,4-dihydroxybenzoic acid (3,4-DHBA) were prepared similarly as done with TNT and were kept in closed vials at least for 2 days prior to sensing experiments. Samples of toluene, methanol, nitrobenzene, benzene, and sodium hydroxide were prepared by wetting a piece of cotton with a small amount of liquid analyte and then the wetted cotton was kept in closed vial. Sensing experiments were performed with all other analytes similarly as done with TNT.

Characterization. Fluorescence spectra of the films were recorded with Varian Eclipse spectrophotometer (excitation wavelength was 340 $\mathrm{nm}$ ). Absorption spectrum of the pyrene doped colloidal ormosil solution was recorded with a UV-vis spectrophotometer (Cary 100, Varian). Surface morphology of the films were investigated with a scanning electron microscope (SEM) (E-SEM, Quanta 200F, FEI) at $10 \mathrm{kV}$, under high vacuum condition after coating a $7 \mathrm{~nm}$ thick goldpalladium layer onto the films. Structure of ormosil colloids were investigated with Transmission Electron Microscope (TEM) (Tecnai G2-F30, FEI) operated at $200 \mathrm{kV}$. Samples were prepared on a holey carbon coated copper grid by dropping $2 \mu \mathrm{L}$ of colloidal ormosil 

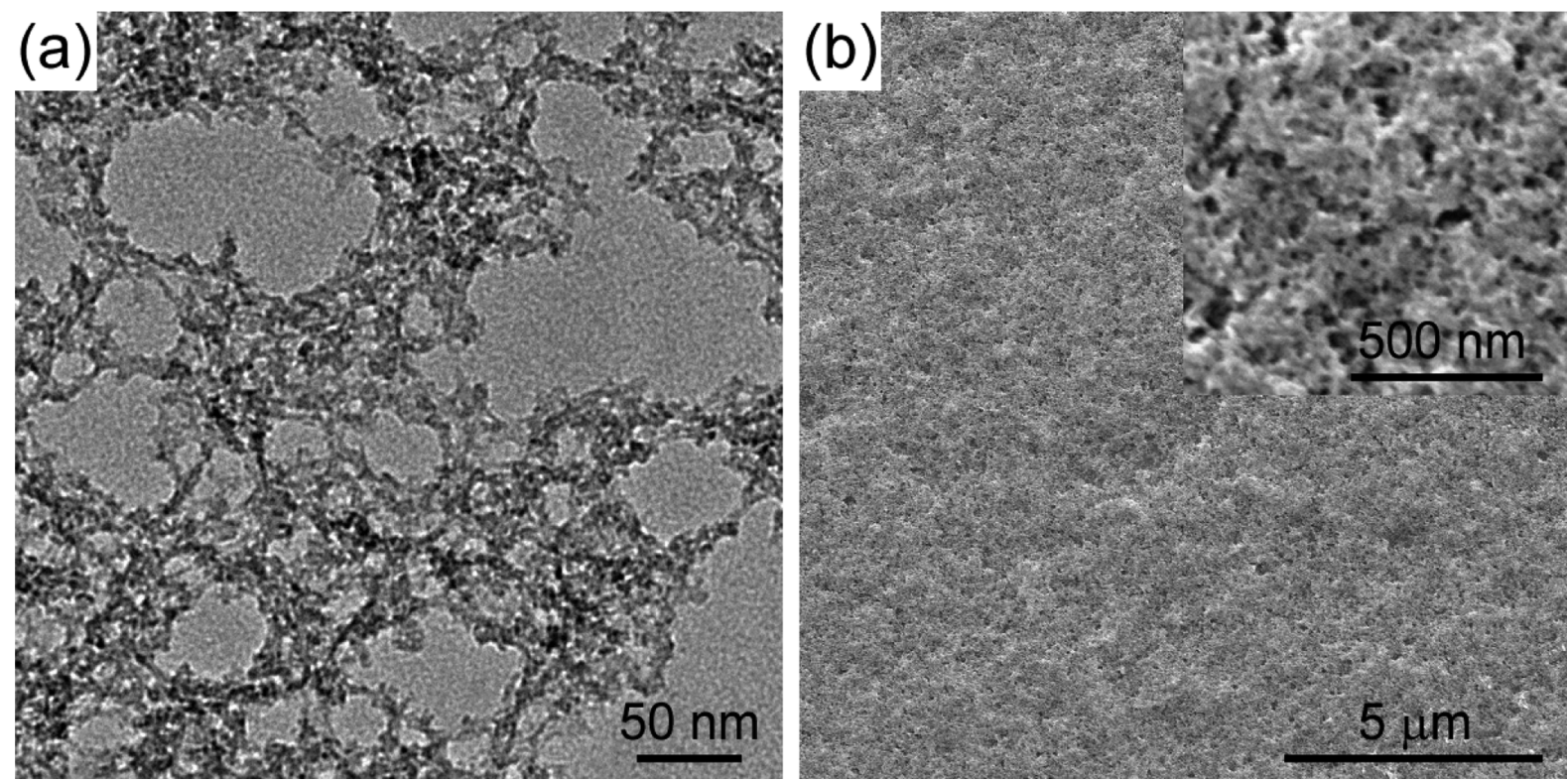

Figure 1. (a) TEM image of the ormosil showing the mesoporous network formed from the interconnected ormosil clusters. (b) SEM image of the pyrene doped mesoporous ormosil thin film. The inset is the higher-magnification SEM image of the same film revealing the highly porous structure.

Table 1. Physical Properties and $I_{\text {exc }} / I_{\text {mon }}$ Ratios of Pyrene Doped Porous and Nonporous Ormosil Thin Films

\begin{tabular}{lcccccc} 
film & thickness $(\mathrm{nm})$ & refractive index $\left(n_{d}\right)$ at $600 \mathrm{~nm}$ & porosity $(\%)$ & water contact angle $(\mathrm{deg})$ & $I_{\text {exc }} / I_{\text {mon }}$ at $0 \mathrm{~h}$ & $I_{\text {exc }} / I_{\text {mon }}$ after $24 \mathrm{~h}$ \\
F1 & 108 & 1.112 & 73.3 & $128.0 \pm 1.6$ & 1.21 & \\
F2 & 251 & 1.166 & 60.5 & $135.7 \pm 1.5$ & 4.09 & 3.43 \\
$\mathrm{~F} 3$ & 1202 & 1.206 & 51 & $145.0 \pm 2.0$ & 0.28 & 0.12 \\
$\mathrm{NPF}^{a}$ & 275 & 1.42 & & $108.1 \pm 0.6$ & & \\
${ }^{a}$ Nonporous film. & & & & & \\
\hline
\end{tabular}

solution. Static water contact angles of the films were measured with a contact angle meter (OCA 30, Dataphysics). A $4 \mu \mathrm{L}$ water droplet was used for each measurement and ten measurements were performed for each film. Laplace-Young fitting was applied to obtain contact angle values. Thickness and refractive indices of the films were determined with an ellipsometer (V-Vase, J.A. Woollam) in the range from 400 to $1000 \mathrm{~nm}$. Cauchy model $\left(n(\lambda)=A+B / \lambda^{2}+C / \lambda^{4}\right)$ was applied to calculate the refractive index and thickness values from the experimental data.

\section{RESULTS AND DISCUSSION}

Preparation of Pyrene-Doped Mesoporous Ormosil Thin Films. We prepared pyrene-doped ormosil thin films by slightly modifying our previous works. ${ }^{27,31,32}$ In the first step, we prepared ormosil gels by using MTMS monomer via a template-free sol-gel method. We dissolved certain amount of pyrene in DMSO and added to the starting reaction solution of the gel. During the gelation, pyrene molecules were physically encapsulated in the porous ormosil network. In the second step, we broke down the gel network by ultrasonication in order to obtain ormosil sol, which is suitable for thin film deposition. In the final step, we coated the ormosil sol onto glass substrates via spin-coating method. For control experiments, we also prepared nonporous ormosil thin films by using MTMS monomer and pyrene.

The structure of pyrene-doped ormosil colloids was investigated with transmission electron microscope (TEM). TEM image shows the mesoporous network, which is formed from interconnected ormosil particles (Figure 1a). Surface morphology of the pyrene-doped mesoporous ormosil thin film was investigated with a scanning electron microscope (SEM).
SEM image shows uniform and highly porous film structure (Figure $1 \mathrm{~b}$ ). The surface of pyrene doped nonporous film was also characterized with SEM. SEM image indicates nonporous, dense and smooth structure of the film (see Figure S1 in the Supporting Information). Thicknesses and refractive indices of the films were measured with a spectroscopic ellipsometer. Thicknesses were found to be 251 and $275 \mathrm{~nm}$ and the refractive indices (at $600 \mathrm{~nm}$ ) were measured as 1.166 and 1.42 for porous and nonporous ormosil films, respectively. Using the measured refractive indices of the films and eq $1\left(n_{\mathrm{p}}, n_{\mathrm{a}}\right.$ and $n_{\mathrm{d}}$ are the refractive indices of porous film, air and nonporous film, respectively), the porosity of the porous ormosil thin film was calculated to be $60.5 \%$.

$$
\text { porosity }=\left(n_{\mathrm{d}}-n_{\mathrm{p}}\right) /\left(n_{\mathrm{d}}-n_{\mathrm{a}}\right)
$$

We also prepared porous ormosil thin films with different thickness by simply changing the concentration of the thin film deposition solution. Reduction in the porosity of the films with increasing thickness was observed that is due to the looser packing of the ormosil colloidal particles in diluted ormosil colloids (Table 1). In addition, surface hydrophobicity of the porous and nonporous films were characterized by measuring the water contact angles of the films. Contact angle values of the coatings was observed to increase (from 128 up to $145^{\circ}$ ) with the increasing thickness, which is due to the difference in the surface topography of the coatings (i.e., surface roughness) (Table 1). ${ }^{31}$ The nonporous film had a lower contact angle value of $108^{\circ}$ than porous films, which is due to its smooth surface structure. 
Absorption spectrum of pyrene in ormosil colloidal solution confirms the existence of pyrene molecules in the ormosil network. Absorption spectrum of pyrene consists of a strong peak at $336 \mathrm{~nm}$ and a weaker peak at $321 \mathrm{~nm}$, which are consistent with the vibration bands of pyrene (Figure 2). ${ }^{4}$ Two

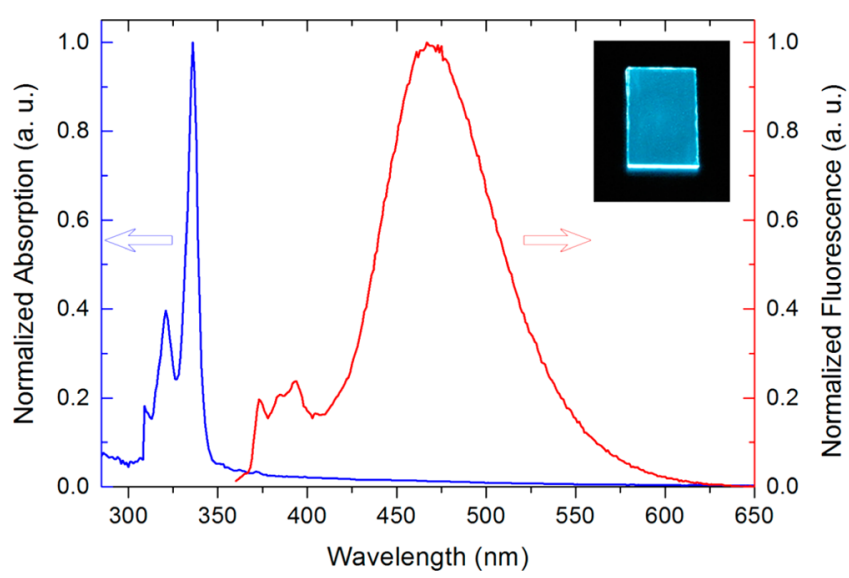

Figure 2. Normalized absorption spectrum (blue) of pyrene-doped ormosil colloid and normalized fluorescence spectrum (red) of pyrenedoped ormosil thin films (Excitation wavelength is $340 \mathrm{~nm}$ ). Absorption spectrum consists of a sharp peak at $336 \mathrm{~nm}$ and a weaker peak at $321 \mathrm{~nm}$. Monomer emission consists of three individual peaks at 373, 385, and $394 \mathrm{~nm}$. Excimer emission is the broad band centered at around $470 \mathrm{~nm}$. The inset shows the photograph of a pyrene-doped ormosil thin film on glass substrate. The photograph was taken under UV-light illumination and the bright blue emission corresponds to pyrene excimer emission.

characteristic emission bands of pyrene were observed in the porous ormosil film (Figure 2). First main band consists of three separate peaks at $373,385,394 \mathrm{~nm}$ indicating the presence of pyrene monomers. Second is the broad band with a fluorescence maximum at around $466-470 \mathrm{~nm}$ and corresponds to the formation of excimers through $\pi-\pi^{*}$ interaction between two pyrene molecules. ${ }^{4}$ Excimer emission of the porous film was very intense compared to monomer emission, suggesting that most of the pyrene molecules were in close proximity, which favored formation of excited dimers. It is important to note that this high excimer to monomer signal ratio was simply achieved by the physical encapsulation of pyrene molecules in the porous ormosil network. Also, the bright blue emission that originated from excimer formation was observed visually under UV light illumination (see the inset in Figure 2).

To investigate pyrene excimer formation conditions in more detail, we prepared several thin films with different pyrene concentrations. As the pyrene concentration increased, both monomer and excimer emission intensities increased (Figure 3a). A weak excimer emission for the film prepared by using ormosil sol with $0.52 \mathrm{mM}$ pyrene was observed due to lack of $\pi-\pi^{*}$ stacking between pyrene molecules in this dilute condition. When pyrene concentration was increased to 2.07 $\mathrm{mM}$ or above, brighter excimer emission was observed. The concentration dependent enhancement of excimer emission signal can be attributed to the decreasing distance in between pyrene molecules at higher concentration and consequently increasing $\pi-\pi^{*}$ stacking possibility between pyrene molecules. We also calculated the ratios of excimer emission intensity to monomer emission intensity $\left(I_{\mathrm{exc}} / I_{\mathrm{mon}}\right)$ for varying pyrene
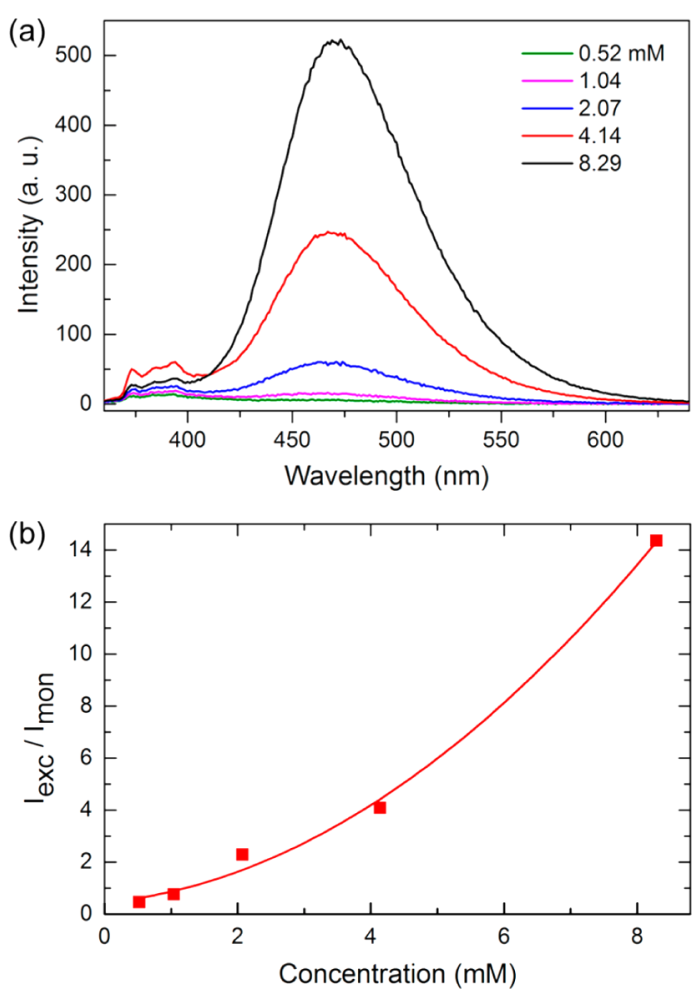

Figure 3. Effect of pyrene concentration on excimer emission intensity. (a) Fluorescence spectra of the films containing different amounts of pyrene. Excimer emission is poor for the films containing 0.52 and $1.04 \mathrm{mM}$ of pyrene. For higher dye concentrations brighter excimer emission is clearly observed. (b) Ratio of the excimer emission intensity to monomer emission intensity $\left(I_{\text {exc }} / I_{\text {mon }}\right)$ with respect to pyrene concentration. (intensities at 470 and $394 \mathrm{~nm}$ in the spectra given in (a) were considered for excimer emission and monomer emission, respectively). There is an exponentially increasing behavior in the $I_{\text {exc }} / I_{\text {mon }}$ ratio with respect to the pyrene concentration.

concentration. It is clear that $I_{\text {exc }} / I_{\text {mon }}$ ratio exponentially increased with increasing pyrene concentration (Figure $3 \mathrm{~b}$ ). The $I_{\text {exc }} / \mathrm{I}_{\text {mon }}$ ratio was 0.46 for the film with $0.52 \mathrm{mM}$ pyrene indicating poor excimer formation. On the other hand, it was 4.09 and 14.34 for the films containing 4.14 and $8.29 \mathrm{mM}$ pyrene, respectively, suggesting that there were sufficient pyrene molecules to closely interact and form excimer emission upon excitation by light. Accordingly, we determined an optimum pyrene concentration to be $4.14 \mathrm{mM}$ in order to obtain bright excimer emission and we used this pyrene concentration in the rest of the study. We also investigated the effect of film thickness on $I_{\text {exc }} / I_{\text {mon }}$ ratio and we observed a reduction in the $I_{\text {exc }} / I_{\text {mon }}$ ratio with decreasing film thickness (Table 1). This was probably due to the increased leakage of pyrene molecules from the ormosil network with the addition of methanol which was used for dilution. In addition, we prepared a nonporous film using the same pyrene concentration as the porous film which contained $4.14 \mathrm{mM}$ pyrene in order to investigate the effect of porous structure on the stability of excimer emission. We did not study the relationship between the $I_{\text {exc }} / I_{\text {mon }}$ ratio and pyrene concentration for the nonporous film because we observed very low and rapidly decaying $I_{\text {exc }} / I_{\text {mon }}$ with these films, which will be discussed below in detail.

Stability of the Excimer Emission. Stability of excimer emission was studied by measuring the emission intensities of 
porous and nonporous films at different time intervals. Fluorescence measurements were conducted immediately after spin-coating and accepted as the $0 \mathrm{~h}$ measurement in which the solvent did not fully left the film. Initially, porous ormosil film demonstrated a bright excimer emission with a high $I_{\text {exc }} / I_{\text {mon }}$ ratio of 4.09 (Figure $4 \mathrm{a}$ ). On the other hand,
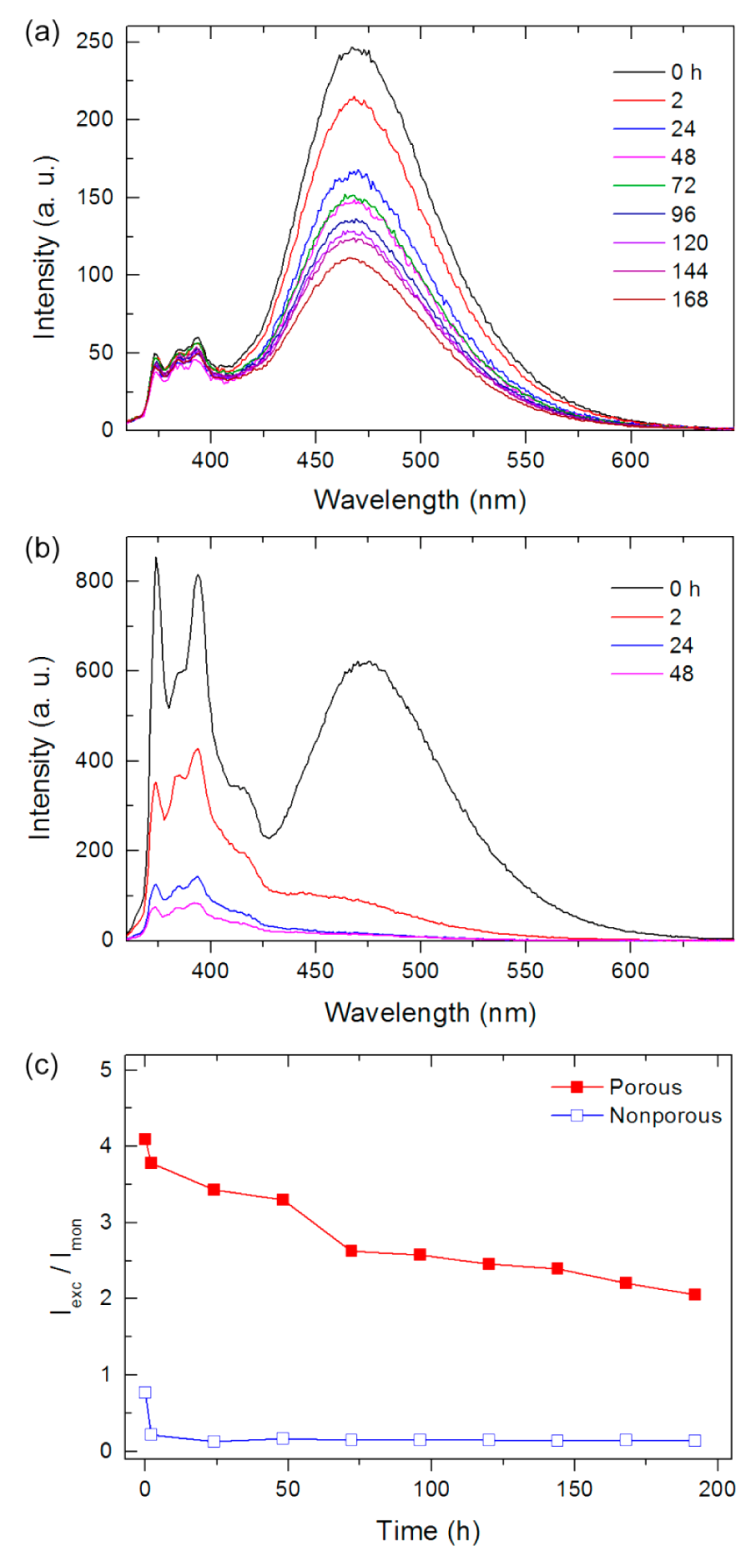

Figure 4. Stability of the pyrene excimer emission in ormosil thin films. (a) Fluorescence spectra of porous film with respect to time. (b) Fluorescence spectra of nonporous film with respect to time. (c) Change in the $I_{\text {exc }} / I_{\text {mon }}$ ratios calculated by the values in the spectra given in a and $b$, for porous and nonporous films, respectively. Porous film has significantly higher $I_{\text {exc }} / I_{\text {mon }}$ value and slower emission decay compared to nonporous film.

nonporous film had a significantly lower $I_{\text {exc }} / I_{\text {mon }}$ ratio of 0.76 (Figure 4b). More importantly, excimer emission intensity of the nonporous film decayed within $2 \mathrm{~h}$. Porous ormosil film, on the other hand, revealed very slow decrease in the excimer emission compared to the nonporous film. Although the excimer emission signal of the porous film decreased gradually with time, it demonstrated very bright excimer emission even after 1 week. Figure $4 \mathrm{c}$ shows the change in the $I_{\text {exc }} / I_{\text {mon }}$ ratio of porous and nonporous films with respect to time. As previously mentioned, the $I_{\text {exc }} / \mathrm{I}_{\text {mon }}$ ratio of the nonporous film decreased rapidly and reached 0.12 within 1 day. The $I_{\text {exc }} / I_{\text {mon }}$ ratio of porous film decreased sharply from 4.09 to 3.78 in the first $2 \mathrm{~h}$ and to 3.43 in 1 day, which may be due to the rapid solvent evaporation from the as-prepared films (Table 1). After $2 \mathrm{~h}$, the decrease in the emission slowed down and reached a constant rate, and then after 1 week, a high $I_{\text {exc }} / I_{\text {mon }}$ ratio of 2.21 was observed for the porous film. Furthermore, we measured a high $I_{\text {exc }} / I_{\text {mon }}$ ratio of 1.6 for a porous film stored at ambient conditions for two months, which indicates the good stability of excimer emission in nanoporous films (see Figure S2 in the Supporting Information). We also studied vacuum drying of the porous films in order to investigate the effect of drying conditions on the excimer emission signal. After spin-coating, we kept one film at $25^{\circ} \mathrm{C}$ in a vacuum oven and another one at room temperature and pressure. We observed that excimer emission of the vacuum-dried film was much lower than that of the ambient-dried film (see Figure S3 in the Supporting Information).

It is believed that hydrophobic and nanoporous ormosil network act as geometrical barrier against the dissociation of pyrene dimers during solvent evaporation and thus greatly promotes excimer formation. It is important to note that investigation of the excimer emission stability of pyrene or its derivatives is missing in most of the previous studies. $14,17,18,34,35$ Herewith, our results show the importance of investigation of time-dependent excimer formation ability in order to produce thin films with long-lived excimer emission signal.

Nitro-explosive Sensing. We analyzed TNT sensing performances of porous films by keeping the films in a closed vial saturated with TNT vapor and measuring their fluorescence intensities after certain time intervals. Fluorescence quenching based TNT sensing mechanism with pyrene is illustrated in Scheme 1. Pyrene-doped ormosil film emits light at around 470 $\mathrm{nm}$ when it is excited at $340 \mathrm{~nm}$ in the absence of TNT. When TNT interacts with pyrene, the excited electron in the pyrene is transferred to the LUMO of the TNT molecule instead of relaxing back to the ground state. As a result, in the presence of TNT, emission of the film is quenched because of the photoinduced electron transfer between TNT and pyrene. ${ }^{13}$

TNT sensing performances of three porous ormosil films with different thicknesses (Table 1) were evaluated. We observed a high fluorescence quenching efficiency for the F1 film against TNT; $32 \%$ after $10 \mathrm{~s}$ and $87.4 \%$ after $5 \mathrm{~min}$ (Figure $5 a)$. On the other hand, for thicker films, the quenching efficiencies were lower; for F2 and F3 (see Figure S4 for F2 and Figure S5 for F3, in the Supporting Information), which were as 18.2 and $8.0 \%$ after $10 \mathrm{~s}$, respectively (Figure $5 \mathrm{~b}$ ). The highest quenching efficiency was obtained with F1, although it had the lowest $I_{\text {exc }} / I_{\text {mon }}$ ratio. The higher quenching efficiency of F1 can be attributed to its higher porosity and lower thickness, which provide a higher TNT diffusion rate.

To compare the decay of pyrene excimer emission with its quenching in the presence of TNT, we calculated 50\% reduction times of initial emission intensities for F2 film. Fifty percent of the initial excimer emission decayed within approximately 6 days (Figure $4 \mathrm{a}$ ). On the other hand, in the presence of TNT, 50\% quenching took only $\sim 150$ s (Figure $5 \mathrm{~b}$ ), which indicates that quenching by TNT was approximately $3 \times 10^{3}$ times faster than decay of the excimer emission. Therefore, pyrene-doped mesoporous ormosil thin films can be 

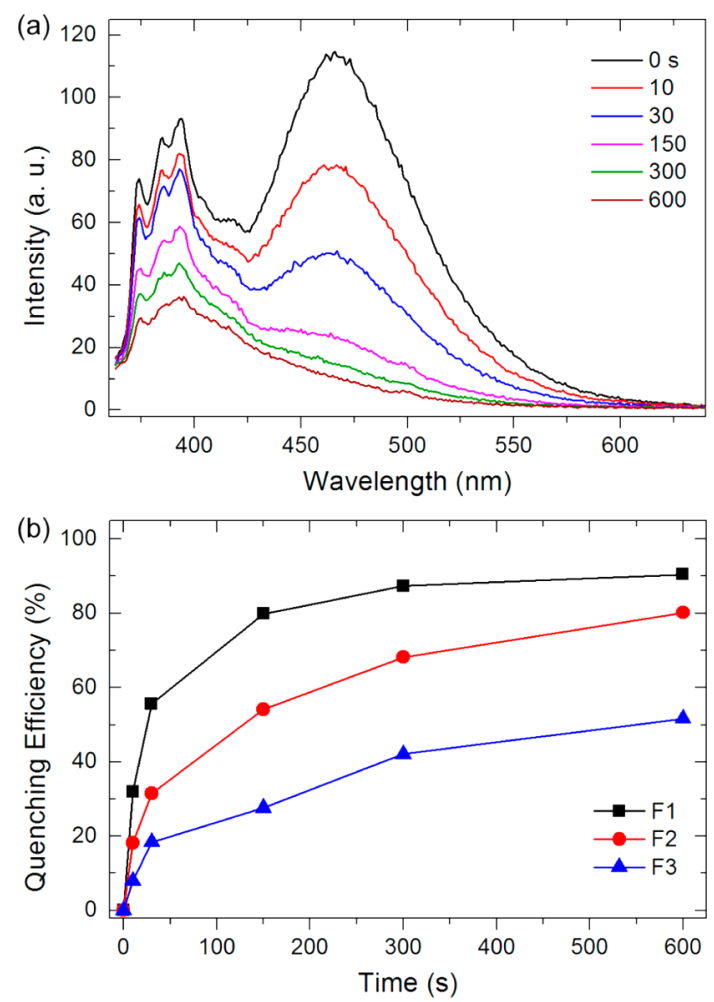

Figure 5. TNT quenching performances of porous ormosil thin films. (a) Time-dependent fluorescence quenching of the F1 film via exposure to TNT, indicating 32 and $87.4 \%$ quenching for $10 \mathrm{~s}$ and 5 min, respectively. (b) Fluorescence quenching efficiencies of the F1, F2, and F3 films for different exposure times. Fluorescence quenching efficiency is depended on the film thickness and F1, the thinnest film, showed the highest quenching efficiency.

used for sensing TNT vapor by monitoring the fast quenching of excimer emission.

In addition, the intense blue excimer emission enabled naked-eye detection of the nitro-explosives. Figure 6 shows

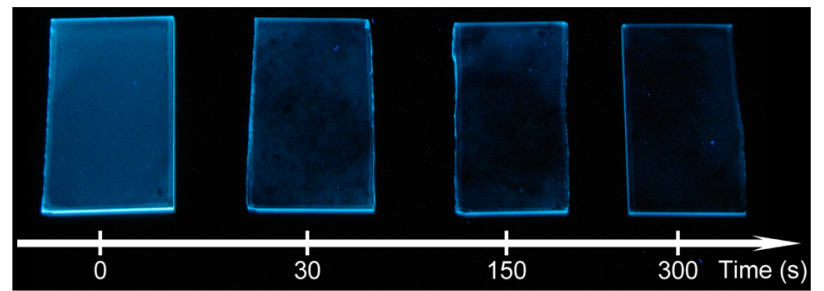

Figure 6. Visual detection of TNT vapor. Photographs taken under UV light illumination show the F1 films, which were exposed to TNT vapor for different durations. A significant quenching in the excimer emission was observed even after $30 \mathrm{~s}$.

emission of F1 films under UV-light illumination after they were exposed to TNT for different durations. The rapid fluorescence quenching of F1 film against TNT vapor can be visually observed from the photographs. Even after exposure to TNT for $30 \mathrm{~s}$, there was a considerable decrease in the bright blue emission of $\mathrm{F} 1$ and after $5 \mathrm{~min}$, almost all emission was quenched.

Selectivity of the Sensor. After demonstrating the sensitivity of the sensor film against TNT vapor, we tested the sensor with other nitroaromatic explosives; DNT and NB, and with also several volatile aromatic and nonaromatic compounds using F2 films (Figure 7). Fluorescence quenching efficiencies of the films against DNT and NB were 39.2 and

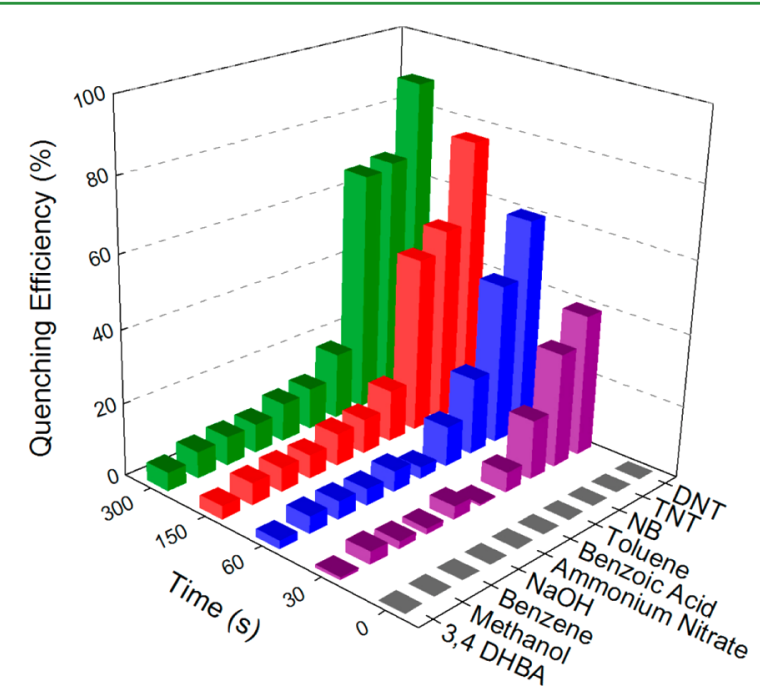

Figure 7. Selectivity of the pyrene-doped porous ormosil films against nitro-explosive vapors. Films have higher quenching efficiency for nitro-explosives compared to various analytes indicating good selectivity of the films. Nitro-explosives largely quenched the fluorescence of F2 films. On the other hand, fluorescence of the films were not quenched significantly by toluene, ammonium nitrate, benzene, benzoic acid, sodium hydroxide, 3,4-DHBA, and methanol.

$16.2 \%$ after $30 \mathrm{~s}$ and 88.2 and $66.7 \%$, after $5 \mathrm{~min}$, respectively. Note that the quenching efficiencies of the F2 film against TNT after $30 \mathrm{~s}$ and $5 \mathrm{~min}$ were 31.5 and $68.2 \%$, respectively (Figure $5 b)$. The higher quenching efficiency of DNT can be attributed to its higher vapor pressure $(100 \mathrm{ppb})$ compared to TNT (5 $\mathrm{ppb})$ at room temperature. ${ }^{33}$ On the other hand, NB showed lower quenching efficiency although its vapor pressure $(\sim 150$ $\mathrm{ppm})$ is considerably higher compared to TNT and DNT. ${ }^{14}$ Besides vapor pressure, the exergonicity of electron transfer between pyrene and nitroaromatic compounds affect the quenching performance of nitroaromatics. ${ }^{24,36} \mathrm{NB}$ has higher LUMO level compared to TNT and DNT due to the lower number of nitro $\left(\mathrm{NO}_{2}\right)$ groups which makes its reduction potential higher and electron accepting ability lower. Orbital energy matching of the LUMO levels of pyrene and NB is not as good as that of pyrene and TNT or DNT. ${ }^{37,38}$ Therefore, NB resulted in a lower quenching efficiency. Lastly, exposure of the sensor to toluene, ammonium nitrate, benzene, benzoic acid, sodium hydroxide, 3,4-DHBA, and methanol did not result in a significant fluorescence quenching, indicating the good selectivity of the sensor toward nitroaromatic explosives.

Reusability of the Sensor. Reusability is an important sensor parameter, which is desired to develop cost-effective sensors. Thus, we examined the regeneration of fluorescence signal of an F2 film after it was quenched with TNT vapor. In order to recover the fluorescence signal, the film was simply immersed in water and washed for $10 \mathrm{~min}$ and then left to dry at room temperature for additional $10 \mathrm{~min}$. In the first recovery step, the fluorescence signal of F2 was not completely recovered (Figure 8a). Because the film was hydrophobic, presumably water could not penetrate through the pores of the film and consequently could not remove all the attached TNT molecules. Nevertheless, there was a significant intensity difference between the quenched and the recovered states 

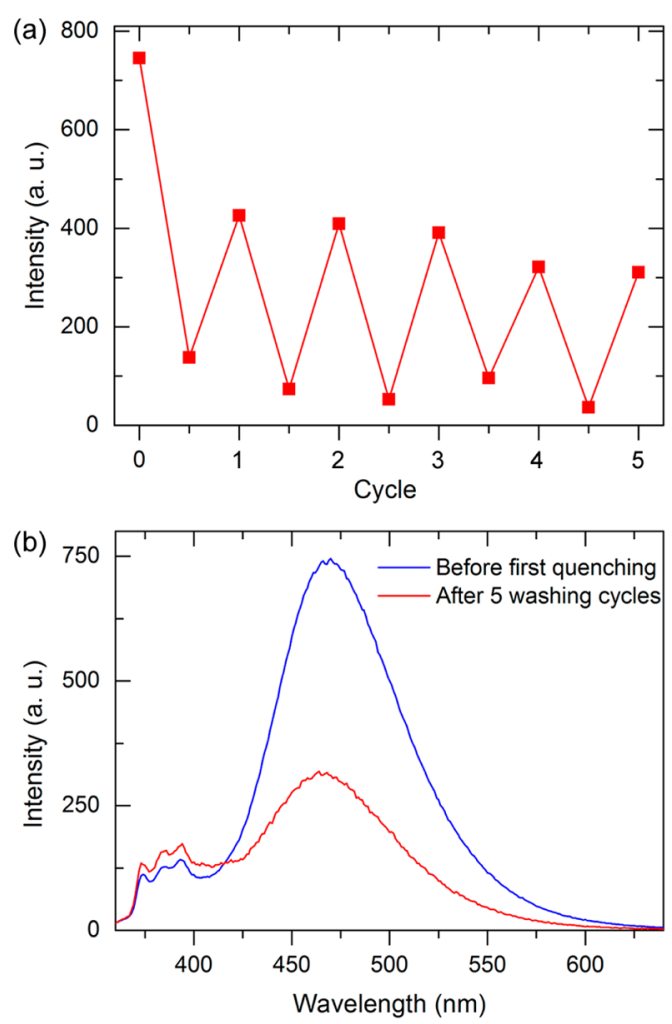

Figure 8. (a) Fluorescence signal quenching and recovery cycles for the F2 film. (b) Fluorescence spectra recorded before the first quenching and after the fifth recovery step of the tested film. The film still has a considerably high excimer emission after the fifth regeneration cycle, which indicates that the film is suitable for further use.

that enables accurate sensing of TNT. Interestingly, after the first recovery cycle, fluorescence of F2 was almost completely recovered for at least four additional cycles. As shown in Figure $8 \mathrm{~b}$, after the fifth cycle, the film still had very strong emission, suggesting the reusability of pyrene-doped ormosil thin film sensors.

\section{CONCLUSIONS}

We described the preparation of porous ormosil thin films with bright and stable pyrene excimer emission via a facile sol-gel process. Excimer emission is stronger and more stable in porous films, whereas it is weak and disappears rapidly in their nonporous counterparts. Excimer emission of nonporous film decays within two hours whereas excimer emission of porous film is stable at least for 2 months. Mesoporous films exhibit rapid fluorescence quenching response against nitro-explosives. More importantly, fluorescence response of the films can be observed by naked eye. Films did not demonstrate any fluorescent quenching when exposed to other various analytes, indicating the good selectivity of the sensor for nitroaromatic explosives. Furthermore, fluorescence signal of the films can be regenerated by simply washing the films with water. We demonstrated that the films can be used for five TNT sensing and signal recovery cycles. We believe that pyrene-doped mesoporous ormosil thin films present a promising sensor platform with their high surface area, easy synthesis, costeffectiveness and reusability.

\section{ASSOCIATED CONTENT}

\section{Supporting Information}

Schematic representation of pyrene-doped ormosil gel preparation and thin film deposition steps. (SEM) image of pyrene doped nonporous film. Fluorescence spectrum of a pyrene doped porous ormosil film stored at ambient conditions for two months. Fluorescence spectra of pyrene doped porous ormosil films dried at ambient pressure and at vacuum. Timedependent fluorescence quenching of the F2 film against TNT vapor. Time-dependent fluorescence quenching of the F3 film against TNT vapor. This material is available free of charge via the Internet at http://pubs.acs.org.

\section{AUTHOR INFORMATION}

\section{Corresponding Author}

*Phone: +90 312290 2513. E-mail: bayindir@nano.org.tr.

\section{Notes}

The authors declare no competing financial interest.

\section{ACKNOWLEDGMENTS}

We would like to thank Hatice Duran, Gokcen Birlik Demirel, Bihter Daglar, and Fahri Emre Ozturk for fruitful discussions and Mustafa Guler for the TEM image. This work is supported by TÜBİTAK under the Project 111T696. M.B. acknowledges partial support from Turkish Academy of Sciences (TUBA).

\section{REFERENCES}

(1) Figueira-Duarte, T. M.; Mullen, K. Pyrene-Based Materials for Organic Electronics. Chem. Rev. 2011, 111, 7260-7314.

(2) Ding, L. P.; Fang, Y. Chemically Assembled Monolayers of Fluorophores as Chemical Sensing Materials. Chem. Soc. Rev. 2010, 39, $4258-4273$

(3) Birks, J. B. Excimers. Rep. Prog. Phys. 1975, 38, 903-974.

(4) Winnik, F. M. Photophysics of Preassociated Pyrenes in Aqueous Polymer Solutions and in Other Organized Media. Chem. Rev. 1993, 93, 587-614.

(5) Fujiwara, Y.; Amao, Y. Optical Oxygen Sensor Based on Controlling the Excimer Formation of Pyrene-1-Butylic Acid Chemisorption Layer onto Nano-porous Anodic Oxidized Aluminium Plate by Myristic Acid. Sens. Actuators, B 2003, 89, 58-61.

(6) Leventis, N.; Elder, I. A.; Rolison, D. R.; Anderson, M. L.; Merzbacher, C. I. Durable Modification of Silica Aerogel Monoliths with Fluorescent 2,7-Diazapyrenium Moieties. Sensing Oxygen near the Speed of Open-Air Diffusion. Chem. Mater. 1999, 11, 2837-2845.

(7) Ueno, A.; Suzuki, I.; Osa, T. Host-Guest Sensory Systems for Detecting Organic Compounds by Pyrene Excimer Fluorescence. Anal. Chem. 1990, 62, 2461-2466.

(8) Huang, J.; Wu, Y.; Chen, Y.; Zhu, Z.; Yang, X.; Yang, C. J.; Wang, K.; Tan, W. Pyrene-Excimer Probes Based on the Hybridization Chain Reaction for the Detection of Nucleic Acids in Complex Biological Fluids. Angew. Chem., Int. Ed. 2011, 50, 401-404.

(9) Yang, R.-H.; Chan, W.-H.; Lee, A. W. M.; Xia, P.-F.; Zhang, H.K.; Li, K. A. A Ratiometric Fluorescent Sensor for $\mathrm{Ag}^{\mathrm{I}}$ with High Selectivity and Sensitivity. J. Am. Chem. Soc. 2003, 125, 2884-2885.

(10) Suzuki, Y.; Morozumi, T.; Nakamura, H.; Shimomura, M.; Hayashita, T.; Bartsh, R. A. New Fluorimetric Alkali and Alkaline Earth Metal Cation Sensors Based on Noncyclic Crown Ethers by Means of Intramolecular Excimer Formation of Pyrene. J. Phys. Chem. B 1998, 102, 7910-7917.

(11) Goodpaster, J. V.; Harrison, J. F.; McGuffin, V. L. Ab Initio Study of Selective Fluorescence Quenching of Polycyclic Aromatic Hydrocarbons. J. Phys. Chem. A 2002, 106, 10645-10654.

(12) Wang, Y.; La, A.; Ding, Y.; Liu, Y.; Lei, Y. Novel SignalAmplifying Fluorescent Nanofibers for Naked-Eye-Based Ultrasensitive Detection of Buried Explosives and Explosive Vapors. Adv. Funct. Mater. 2012, 22, 3547-3555. 
(13) Demirel, G. B.; Daglar, B.; Bayindir, M. Extremely Fast and Highly Selective Detection of Nitroaromatic Explosive Vapours Using Fluorescent Polymer Thin Films. Chem. Commun. 2013, 49, 61406142 .

(14) Bai, H.; Li, C.; Shi, G. Pyrenyl Excimers Induced by the Crystallization of POSS Moieties: Spectroscopic Studies and Sensing Applications. ChemPhysChem 2008, 9, 1908-1913.

(15) Basabe-Desmonts, L.; Reinhoudt, D. N.; Crego-Calama, M. Design of Fluorescent Materials for Chemical Sensing. Chem. Soc. Rev. 2007, 36, 993-1017.

(16) Li, M.-C.; Ho, R.-M.; Lee, Y.-D. Photo-induced Excimer Formation of Pyrene-labeled Polymers for Optical Recording. J. Mater. Chem. 2011, 21, 2451-2454.

(17) Pujari, S. R.; Kambale, M. D.; Bhosale, P. N.; Rao, P. M. R.; Patil, S. R. Optical Properties of Pyrene Doped Polymer Thin Films. Mater. Res. Bull. 2002, 37, 1641-1649.

(18) Jang, H.-S.; Wang, Y.; Lei, Y.; Nieh, M.-P. Controllable Formation of Pyrene $\left(\mathrm{C}_{16} \mathrm{H}_{10}\right)$ Excimers in Polystyrene/Tetrabutylammonium Hexafluorophosphate Films through Solvent Vapor and Temperature Annealing. J. Phys. Chem. C 2013, 117, 1428-1435.

(19) Kaufman, V. R.; Avnir, D. Structural Changes along the Sol-GelXerogel Transition in Silica As Probed by Pyrene Excited-State Emission. Langmuir 1986, 2, 717-722.

(20) Keeling-Tucker, T.; Brennan, J. D. Fluorescent Probes as Reporters on the Local Structure and Dynamics in Sol-Gel-Derived Nanocomposite Materials. Chem. Mater. 2001, 13, 3331-3350.

(21) Dunn, B.; Zink, J. I. Probes of Pore Environment and MoleculeMatrix Interactions in Sol-Gel Materials. Chem. Mater. 1997, 9, 22802291.

(22) Deng, Q.; Hu, Y.; Moore, R. B.; McCormick, C. L.; Mauritz, K. A. Nafion/ORMOSIL Hybrids via in Situ Sol-Gel Reactions. 3. Pyrene Fluorescence Probe Investigations of Nanoscale Environment. Chem. Mater. 1997, 9, 36-44.

(23) Tao, S.; Li, G.; Zhu, H. Metalloporphyrins As Sensing Elements for the Rapid Detection of Trace TNT Vapor. J. Mater. Chem. 2006, 16, 4521-4528.

(24) Tao, S.; Yin, J.; Li, G. High-performance TNT Chemosensory Materials Based on Nanocomposites with Bimodal Porous Structures. J. Mater. Chem. 2008, 18, 4872-4878.

(25) Long, Y.; Chen, H.; Yang, Y.; Wang, H.; Yang, Y.; Li, N.; Li, K.; Pei, J.; Liu, F. Electrospun Nanofibrous Film Doped with a Conjugated Polymer for DNT Fluorescence Sensor. Macromolecules 2009, 42, 6501-6509.

(26) Leventis, N.; Rawashdeh, A.-M. M.; Elder, I. A.; Yang, J.; Dass, A.; Sotiriou-Leventis, C. Synthesis and Characterization of $\mathrm{Ru}(\mathrm{II})$ Tris(1,10-phenanthroline)-Electron Acceptor Dyads Incorporating the 4-Benzoyl- $N$-methylpyridinium Cation or $N$-Benzyl- $N^{\prime}$-methyl Viologen. Improving the Dynamic Range, Sensitivity, and Response Time of Sol-Gel-Based Optical Oxygen Sensors. Chem. Mater. 2004, 16, $1493-1506$

(27) Yildirim, A.; Budunoglu, H.; Deniz, H.; Guler, M. O.; Bayindir, M. Template-Free Synthesis of Organically Modified Silica Mesoporous Thin Films for TNT Sensing. ACS Appl. Mater. Interfaces 2010, 2, 2892-2897.

(28) Tozuka, Y.; Yonemochi, E.; Oguchi, T.; Yamamoto, K. Fluorometric Studies of Pyrene Adsorption on Porous Crystalline Cellulose. J. Colloid Interface Sci. 1998, 205, 510-515.

(29) Dabestani, R.; Kidder, M.; Buchanan, A. C., III Pore Size Effect on the Dynamics of Excimer Formation for Chemically Attached Pyrene on Various Silica Surfaces. J. Phys. Chem. C 2008, 112, 1146811475 .

(30) Bhagat, S. D.; Oh, C. S.; Kim, Y. H.; Ahn, Y. S.; Yeo, J. G. Methyltrimethoxysilane Based Monolithic Silica Aerogels via Ambient Pressure Drying. Microporous Mesoporous Mater. 2007, 100, 350-355.

(31) Budunoglu, H.; Yildirim, A.; Guler, M. O.; Bayindir, M. Highly Transparent, Flexible, and Thermally Stable Superhydrophobic ORMOSIL Aerogel Thin Films. ACS Appl. Mater. Interfaces 2011, 3, 539-545.
(32) Yildirim, A.; Budunoglu, H.; Yaman, M.; Guler, M. O.; Bayindir, M. Template Free Preparation of Nanoporous Organically Modified Silica Thin Films on Flexible Substrates. J. Mater. Chem. 2011, 21, 14830-14837.

(33) Rose, A.; Zhu, Z. G.; Madigan, C. F.; Swager, T. M.; Bulovic, V. Sensitivity Gains in Chemosensing by Lasing Action in Organic Polymers. Nature 2005, 434, 876-879.

(34) Sluch, M. I.; Vitukhnovsky, A. G.; Petty, M. C. Pyrene Excimer Formation in Langmuir-Blodgett Films. Thin Solid Films 1996, 284285, 622-626.

(35) Kokado, K.; Iwamura, T.; Chujo, Y. Synthesis and Photoluminescence Properties of Pyrene-Incorporated Organic-Inorganic Polymer Hybrids. Polym. J. 2008, 40, 402-408.

(36) Yang, J.-S.; Swager, T. M. Fluorescent Porous Polymer Films as TNT Chemosensors: Electronic and Structural Effects. J. Am. Chem. Soc. 1998, 120, 11864-11873.

(37) Toal, S. J.; Trogler, W. C. Polymer Sensors for Nitroaromatic Explosives Detection. J. Mater. Chem. 2006, 16, 2871-2883.

(38) Salinas, Y.; Martínez-Máñez, R.; Marcos, M. D.; Sancenón, F.; Costero, A. M.; Parra, M.; Gil, S. Optical Chemosensors and Reagents to Detect Explosives. Chem. Soc. Rev. 2012, 41, 1261-1296. 